\title{
CAN BLACK FORCE A WIN?
}

\author{
David Dowe \\ Department of Information Systems \\ Swinburne, Australia
}

It is commonly believed that, with correct play, White can force at least a draw from the game's initial position. In the hope of one day seeing a (computer-aided) proof, we assume the role of the devil's advocate and ask whether White might be in zugzwang and hence Black might have a forced win in the game's initial position. For curiosity, we provide two examples below of symmetrical and legal positions in which White is in zugzwang and Black can force a win.

Let us define a position on a chess-board to be symmetrical if the following conditions (i)-(v) hold:

(i) the configuration of the pieces is symmetrical, i.e., moving pieces from their current squares to the square(s) obtained by a reflection about the line (the " $4.5^{\text {th }}$ rank) along the middle of the board, and then changing the colours of all pieces (Black to White and White to Black) restores the pieces to their original configuration*;

(ii) either both Kings are eligible to castle Kingside (possibly later on) or neither King is;

(iii) either both Kings are eligible to castle Queenside (possibly later on) or neither King is;

(iv) no Pawn can be captured "en passant";

(v) it is White's move.

We further say that a position is symmetrical and legal if it is both symmetrical and

(vi) it can be obtained from the initial position via some legal sequence of moves.

(Note that the sequence of moves need not follow symmetrical play).

It follows from (i) and (vi) that neither King is currently in check.

Diagrams 1 and 2 below are both examples of symmetrical and legal positions from which Black has a forced win.

The position in Diagram 1 below can be reached from the initial position by the following sequence of 21 moves:

1. e4 e5 2. d4 d5 3. dxe5 dxe4 4. Qd5 b5 5. Qxa8 Qd4 6. b4 Qxa1 7. h4 h5 8. Bf4 Bf5 9. Bh2 Bh7 10. Be2 Be7 11. Bg4 Bg5 12. Nf3 Nf6 13. g3 g6 14. Kf1 Qxb1+ 15. Kg2 Qg1+ 16. Bxg1 Kf8 17. Qxb8+ Kg7 18. Qg8+ Bxg8 19. Kh2 Kh7 20. hxg5 hxg4 21. exf6 exf3

Note that giving White a Rook at g2 and Black a Rook at g7 in Diagram 1 leaves the position legal and symmetrical, and White is still in zugzwang.

Note further that the above comments all still apply if we replace the white Rook at h1 and the black Rook at h8 respectively by a white Knight and a black Knight.

* This condition is sufficient to ensure that the configuration of the pieces is symmetrical, but we need some other conditions to ensure that the position is symmetrical (and that neither team is disadvantaged). 
The position in Diagram 2 below can be reached from the initial position by the following sequence of 21 moves:

1. d4 d5 2. e4 e5 3. f4 f5 4. g4 g5 5. fxg5 fxg4 6. dxe5 dxe4 7. Qd5 g3 8. Qxg8 Qd4 9. Qxh8 Qxg1 10. Qxh7 Qxh1 11. Qxc7 Qxh2 12. Qxb7 Qxc2 13. Qxa7 Qxb2 14. Qxa8 Qxa2 15. Qxb8 Qxa1 16. Qxc8+ Ke7 17. Qxf8+ Kxf8 18. g6 Qxb1 19. e6 Qxc1+ 20. Ke2 Qxf1+21. Kxf1 e3

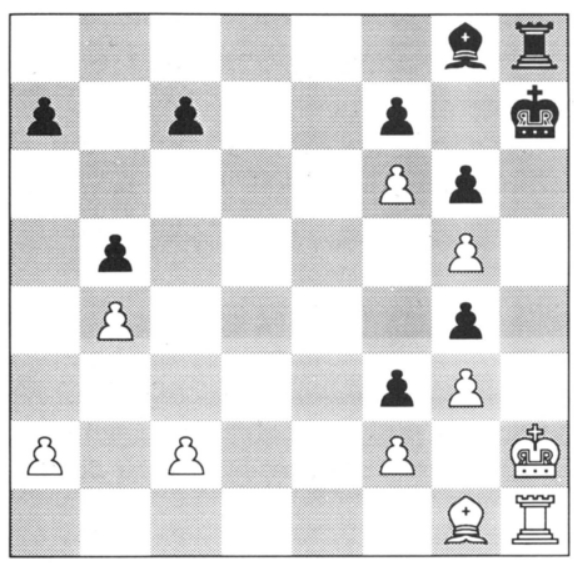

DIAGRAM 1 White to move.

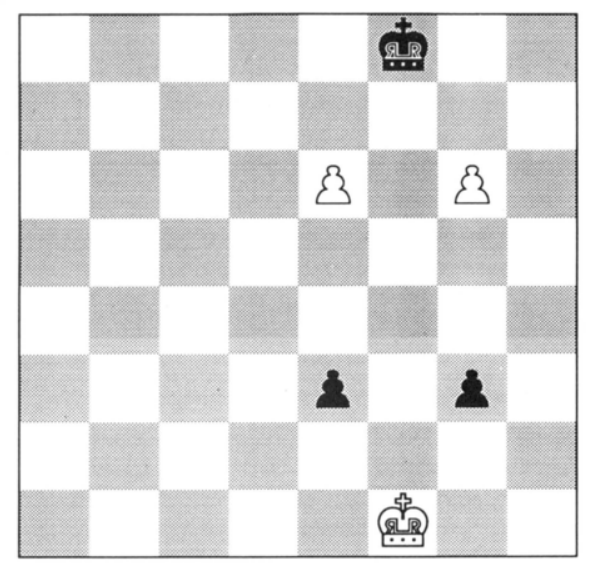

DIAGRAM 2 White to move.

We note that in the position in Diagram 1 both sides have 7 Pawns and a total of 10 pieces each. (Note that we can get up to 12 pieces (and 8 Pawns) each by placing a white Rook at g2 and a Black Rook at g7, and White and Black pawns at $\mathrm{d} 4$ and $\mathrm{d} 5$ respectively).

We note that in the position in Diagram 2 both sides have 2 Pawns and a total of 3 pieces each. (This would appear to be the minimum possible).

Note also that if we were to insist on having at least one piece each other than King and Pawn(s), then we could do this using 6 Pawns each by modifying Diagram 2 to include white and black Knights at a1 and a8 respectively, additional white Pawns at b3, b5, c2 and c6; additional black Pawns at b4, b6, c3 and c7.

In light of the above observations, we ask the following questions about symmetrical and legal positions from which Black has a forced win:

Question 1: Can one be obtained in less than 21 moves each?

Question 2: Can it be done with more than 12 pieces each?

Question 3: Can it be done with less than 3 pieces each?

Question 4: If we use at least one piece other than King and Pawn(s), can it be done with less than 6 Pawns each?

Note that a negative answer to either Question 1 or Question 2 would imply an affirmative answer to Question 5 .

Question 5: Is there any way of showing that Black cannot, with correct play, force a win from the game's initial position against any white defence?

\section{Symmetrical Play}

Mr. Jules Welling (Best, The Netherlands) has shown a way of obtaining the position in Diagram 1 in 23 moves with symmetrical play. He has also referred me to "Sam Loyd and his chess problems" by Alain C. White (Dover, New York), which contains the first problems in Mirror Chess, and has drawn my attention to the following Loyd (1866) game from p. 62 of Alain White's book: 1. e4 e5 2. Ke2 Ke7 3. Ke3 Ke6 4. Qf3 Qf6 5. Ne2 Ne7 6. b3 b6 7. Ba3 Ba6 8. Nd4+ exd4 mate. The play in this game is symmetrical until the end of move 7, and Black's only legal $8^{\text {th }}$ move is to checkmate White.

My question is whether all symmetrical and legal positions can be obtained with symmetrical play. I conjecture that the answer is "Yes". Computer solutions are invited. 